\title{
TESTE DE QUANTIFICAÇÃO DE ETANOL PARA AVALIAÇÃO DA QUALIDADE FISIOLÓGICA SEMENTES DE QUINOA
}

Rafael Oliveira Vergara, Vanessa Nogueira Soares, Raimunda Nonata Oliveira da Silva, Andrea Bicca Noguez Martins, Gizele Ingrid Gadotti, Francisco Amaral Villela

Universidade Federal de Pelotas - UFPel. E-mail: agrorafaelvergara@gmail.com

\section{RESUMO}

A quinoa é uma cultura de origem andina. Atualmente apresenta grande demanda pelo mercado consumidor devido a qualidade nutricional de seus grãos. Assim, a importância do conhecimento do potencial fisiológico de sementes de quinoa ganha destaque. Uma metodologia inovadora, sendo considerado um teste rápido para avaliação do nível de vigor é o teste do etanol, desta forma, objetivou-se estudar a relação entre a produção de etanol e o desempenho fisiológico de sementes de quinoa. Foram utilizados quatro lotes de sementes, com distintos níveis de vigor e três diferentes massas de sementes $(1,0 ; 1,5$ e 2,0 g). Posteriormente, as sementes foram imersas em água por um período de 24 horas a uma temperatura constante de $40^{\circ} \mathrm{C}$, após este período, foi efetuada a quantificação de emissão de etanol. $\mathrm{O}$ teste foi eficiente para diferir os níveis de vigor dos lotes, sendo que em 1,0 g é obtido a mesma distinção que a oferecida por métodos tradicionais (emergência e índice de velocidade de emergência) com menor tempo de resposta.

Palavras-chave: Chenopodium quinoa Willd; controle de qualidade; pseudocereal; vigor.

\section{ETHANOL QUANTIFICATION TEST TO EVALUATE THE PHYSIOLOGICAL QUALITY OF QUINOA SEEDS}

\begin{abstract}
Quinoa is a crop originated in the Andean region. Currently, consumer demand rises due to the nutritional quality of it grains. Thus, to know the importance of physiological potential of quinoa seeds is highlighted. The ethanol test is an innovative methodology it is considered a quick test to evaluate seed vigor level. This study aimed to evaluate the physiological quality of quinoa seeds by the ethanol test. We used four quinoa seeds lots with different vigor levels and three weight of seeds (1.0, 1.5, and $2.0 \mathrm{~g})$. Seeds were kept underwater for 24 hours at a temperature of $40^{\circ} \mathrm{C}$. After this period, the quantification of ethanol produced by quinoa seeds was performed. It was observed that the ethanol test is effective to differ the seed lots vigor levels. Similar results achieved by traditional methods (seedling emergence, emergence speed index) are found using $1.0 \mathrm{~g}$ of quinoa seeds in a fast response.
\end{abstract}

Keywords: Chenopodium quinoa Willd; quality control; seed production; pseudocereal; vigor.

\section{INTRODUÇÃO}

A quinoa (Chenopodium quinoa Willd), pertence à família Chenopodiaceae, uma planta de ciclo anual, originária do Planalto Boliviano, mais precisamente da Cordilheira dos Andes, foi bastante cultivada pelos Incas, e por outros povos que habitavam a região (SPEHAR, 2007).

A principal forma de propagação de quinoa é via semente. Suas sementes são constituídas por episperma (tecido que cobre a semente), perisperma (tecido de reserva) e pelo embrião, este por sua vez formado pelos cotilédones, hipocótilo e radícula (CARVALHO; NAKAGAWA, 2012). O perisperma representa o principal tecido de reserva nutricional, correspondente, pela proximidade em composição organo-mineral, ao endosperma nas sementes de cereais. No entanto, não pertencente à mesma família botânica, sendo a quinoa é frequentemente referida como um "pseudocereal" (SPEHAR, 2002). Para a comercialização das sementes tem-se a necessidade de testes padronizados de germinação e pureza física. A padronização de 
testes para avaliação da qualidade de sementes de quinoa é importante, por ser tratar de uma espécie de cultivo recente no Brasil e com futuro promissor, levando em conta a expansão do mercado consumidor.

A avaliação do potencial fisiológico de sementes é o principal componente de um programa de controle de qualidade, visto que fornece informações que identificam e solucionam problemas durante $o$ processo produtivo, além de estimar o desempenho das sementes em campo (MARTINS et al., 2014). Quando falamos de testes para avaliação da qualidade fisiológica de sementes para fins de comercialização e semeadura, podemos nos concentrar no teste de germinação, que é realizado em condições ideais e artificiais, permitindo a expressão da máxima qualidade das sementes. No entanto, este teste apresenta limitações, principalmente, no que se refere à diferenciação de lotes e a relativa demora na obtenção dos resultados, o que tem estimulado, ao longo dos anos, o desenvolvimento de testes de vigor que sejam confiáveis e rápidos, agilizando as decisões (BERTOLIN et al., 2011) e complementando as informações fornecidas pelo teste de germinação.

Sendo assim, a identificação de testes de vigor que forneçam margem segura quanto ao comportamento das sementes em campo vem sendo uma busca incansável e uma necessidade, visto que as condições adversas do ambiente impõem desuniformidade entre o teste de germinação e os resultados de campo, estabelecendo assim a necessidade de identificar testes que forneçam condições equiparadas à germinação em campo, aliado a todas as adversidades que possam afetar o desempenho de uma cultivar (MARTINS et al., 2014).

As alterações no metabolismo do etanol são indicadores da deterioração em sementes. Essas mudanças no metabolismo do etanol se apresentam provavelmente relacionadas a um declínio na função mitocondrial resultante da oxidação da membrana, que por sua vez ocasiona na menor produção de NAD+ e também limitando a capacidade de degradar o etanol (KODDE; GROOTE, 2015). Estudando o vigor em sementes de canola, Buckley e Huang (2011) observaram a ocorrência de interação significativa entre a produção de etanol e o vigor de sementes. No mesmo estudo, foi evidenciado o teste de emissão de etanol como um teste rápido e simples, por não necessitar de nenhum tipo de instalação especial.

Neste sentido, Woodstock e Taylorson (1981), em estudo relacionado à produção de acetaldeido e etanol em sementes de soja, verificaram correlação significativa, indicando que o nível de produção destes compostos estava relacionados à deterioração das sementes, sendo atribuído a alterações nas membranas mitocondriais.

O objetivo deste trabalho foi estudar a relação entre a produção de etanol e a massa de sementes de quinoa, e sua correlação com desempenho fisiológico.

MATERIAL E MÉTODOS

O trabalho foi conduzido no Laboratório Didático de Análise de Sementes Flávio Farias da Rocha da Universidade Federal de Pelotas, durante o ano de 2015. Foram utilizadas sementes de quinoa (Chenopodium quinoa Willd) oriundas de quatro lotes da cultivar BRS Piabiru, com germinação semelhante, produzidas no ano de 2015.

Para a avaliação da qualidade fisiológica inicial das sementes foram conduzidos os seguintes teste:

Germinação: foram utilizadas quatro repetições com duzentas sementes cada, semeadas em caixas gerbox sobre duas folhas papel mata borrão, previamente umedecido com duas vezes o seu peso $(\mathrm{g})$ seco em volume de água $(\mathrm{mL})$. Foi contabilizado o número de plântulas normais ao final de sete dias, sendo os resultados expressos em percentual de plântulas normais (BORGES et al., 2015).

Emergência em canteiro: foram semeadas cinco repetições de 100 sementes cada, em canteiro. A avaliação foi efetuada sete dias após semeadura e resultado expresso em percentual de plântulas emergidas.

Índice de velocidade de emergência: conduzido conjuntamente com o teste de emergência em campo. Os dados de contagem do número de plântulas emergidas, coletados diariamente foram utilizados para determinar o índice de velocidade de emergência, conforme a fórmula proposta por Maguire (1962).

Teste do etanol: quantidades de sementes dos quatro lotes foram acondicionadas em garrafas PET de $500 \mathrm{~mL}$, imersas em $20 \mathrm{~mL}$ de água destilada, o suficiente para as sementes em estado de hipoxia. As garrafas foram fechadas com silicone e incubadas a $40{ }^{\circ} \mathrm{C}$ por 24 horas. A concentração de etanol foi mensurada com a 
utilização de um etilômetro modelo Instrutherm BFD-60 modificado. Previamente, foram realizados pré-testes para verificar o tempo necessário para leitura. Foram utilizados três diferentes massas de sementes $(1,0 ; 1,5$ e $2,0 \mathrm{~g})$, estabelecendo a quantidade de sementes mais adequada para condução do teste.

$O$ delineamento experimental utilizado foi inteiramente casualizado, com cinco repetições. Os dos dados foram submetidos a análise de variância pelo teste $F$ a $5 \%$ de probabilidade de erro e, quando significativo, as médias foram comparadas utilizando o teste de Tukey a $5 \%$ de probabilidade. Também foi realizada análise de correlação de Pearson entre os componentes principais a $1 \%$ de probabilidade. Para efetuar as análises foi utilizado o programa estatístico Winstat.

RESULTADOS E DISCUSSÃO

Os lotes tinham germinação semelhante, entretanto distintos níveis de vigor (Tabela 1), pelo fato de ser importante e coerente a comparação do vigor de lotes de sementes com germinação semelhante (MARCOS FILHO, 1999). Estes apresentaram três níveis de vigor, fato é evidenciado nas pelo índice de velocidade de emergência e pela emergência em campo.

Tabela 1. Germinação (G), índice de velocidade de emergência (IVE), emergência (E), quantificação de etanol (QE) utilizando diferentes massas de sementes em quatro lotes de quinoa.

\begin{tabular}{lllllll}
\hline Lote & $\mathrm{G}$ & IVE & $\mathrm{E}$ & QE $(1 \mathrm{~g})$ & QE $(1,5 \mathrm{~g})$ & QE (2g) \\
\hline 1 & $92 \mathrm{a}$ & $63,55 \mathrm{a}$ & $80 \mathrm{a}$ & $0,130 \mathrm{c}$ & $0,207 \mathrm{c}$ & $0,310 \mathrm{~b}$ \\
2 & $92 \mathrm{a}$ & $61,74 \mathrm{a}$ & $81 \mathrm{a}$ & $0,124 \mathrm{c}$ & $0,400 \mathrm{a}$ & $0,440 \mathrm{a}$ \\
3 & $92 \mathrm{a}$ & $39,96 \mathrm{~b}$ & $68 \mathrm{~b}$ & $0,430 \mathrm{~b}$ & $0,310 \mathrm{~b}$ & $0,290 \mathrm{~b}$ \\
4 & $88 \mathrm{a}$ & $31,10 \mathrm{c}$ & $49 \mathrm{c}$ & $0,570 \mathrm{a}$ & $0,281 \mathrm{bc}$ & $0,310 \mathrm{~b}$ \\
\hline $\mathrm{CV}(\%)$ & 2,13 & 8,35 & 9,69 & 10,58 & 21,86 & 22,86 \\
\hline
\end{tabular}

Médias seguidas pela mesma letra na coluna não diferem entre si, pelo teste Tukey, a 5\% de probabilidade.

A utilização de três diferentes massas de sementes (Tabela 1) evidenciou que, para a quinoa, a massa de sementes se torna um fator que exerce influência sobre a eficiência do teste em estudo. De tal forma, que a utilização de massa de sementes inadequada pode comprometer o resultado obtido no teste em estudo.

Entretanto, ao utilizar massa de sementes de 1,0g (Tabela 1), ocorreu a separação dos lotes em três níveis de vigor. A diferença na produção de etanol demonstrou a variação dos lotes quanto ao vigor, ou seja, lotes menos vigorosos apresentam maior produção do subproduto. Vale ressaltar que existiu correspondência quanto à diferenciação dos lotes entre testes de vigor (emergência em campo e índice de velocidade de emergência) e o resultado encontrado no teste de quantificação do etanol.

A utilização de $1,0 \mathrm{~g}$ de sementes no teste do etanol (Tabela 1), apresentando elevado coeficiente de variação. A produção de etanol ao utilizar 1,5 g de sementes, não mostrou associação com os níveis de vigor encontrados na emergência em campo e no índice de velocidade de emergência, apresentando estes testes reconhecida eficiência, sendo utilizados na avaliação de vigor em espécies como girassol (SÁ BRAZ; ROSSETO, 2009), pepino (TORRES et al., 2012) e soja (WENDT et al., 2017).

Utilizando 2,0 g de sementes (Tabela 1), o efeito foi semelhante ao indicado na utilização de $1,5 \mathrm{~g}$ de sementes. Assim, não ocorreu associação entre a produção de etanol e o nível de vigor dos lotes de sementes de quinoa.

Pela análise de correlação de Pearson (Tabela 2), fica evidente a existência de correlação entre as variáveis indice de velocidade de emergencia, emergencia e produção de etanol - Demonstrando a eficiência do teste de quantificação do etanol na avaliação do vigor dos lotes de sementes de quinoa. 
Tabela 2. Correlação de Pearson entre as variáveis emergência (E), índice de velocidade de emergência (IVE) e produção de etanol (PE), com nível de $1 \%$ de significância

\begin{tabular}{lllll}
\hline & $\mathrm{E}$ & $\mathrm{PE}(1,0 \mathrm{~g})$ & $\mathrm{PE}(1,5 \mathrm{~g})$ & $\mathrm{PE}(2,0 \mathrm{~g})$ \\
\hline IVE & $0.6985^{*}$ & $-0.9517^{*}$ & $0,0356^{\mathrm{ns}}$ & $-0,0109^{\text {ns }}$ \\
$\mathrm{E}$ & - & $-0.8421^{*}$ & $-0,2247^{\text {ns }}$ & $0,4034^{\text {ns }}$ \\
\hline
\end{tabular}

*Significativo em nível de $1 \%$.

Observando a produção de etanol empregando $1,0 \mathrm{~g}$ de sementes e o índice de velocidade de emergência (Tabela 2), nota-se a existência de correlação negativa entre as variáveis $(-0,9517)$. O aumento na produção de etanol correlaciona-se negativamente com 0 índice de velocidade de emergência, sendo este um importante parâmetro na avaliação de vigor de lotes de sementes, de acordo com Menezes et al. (2007), a emergência a campo ratifica, complementa e auxilia na definição do potencial fisiológico, pois estima o desempenho de sementes e lotes em condições variadas de ambiente. Vale ressaltar que valores elevados na produção de etanol indicam sementes menos vigorosas, visto que ocorre maior formação de etanol em sementes, conforme o avanço no processo de deterioração (CAVALCANTE et al., 2017).

A existência de correlação entre a produção de etanol e a emergência de plântulas em campo (Tabela 2) é significativa $(-0,8421)$. Assim analisando um determinado lote, o aumento na porcentagem de plântulas emergidas em condição de campo representa a diminuição na produção de etanol. Sendo o teste de emergência em campo um dos mais importantes testes de vigor, pois a manifestação do vigor em sementes é o resultado da interação das características que determinam seu potencial para uma rápida e uniforme emergência de plântulas a campo (BAALBAKI et al., 2009). Deste modo, pela associação observada entre produção de etanol e emergência de plântulas a campo, é possível inferir que o teste de quantificação de etanol é capaz de estimar o vigor em lotes de sementes de quinoa.

Os resultados obtidos neste trabalho corroboram com o resultado apresentado por Kodde et al. (2012), ao observarem a existência de correlação negativa entre a produção de etanol e a qualidade fisiológica de sementes de repolho. Ainda de acordo com os autores, na espécie em questão, o aumento na produção de etanol ocorreu em sementes que foram armazenadas em condição ambiental, submetidas a estresse ou em sementes imaturas. Vale destacar que os autores evidenciam que a falta de oxigênio não é o principal gatilho para a produção de etanol, pois a produção de etanol ocorreu simultaneamente ao consumo de oxigênio.

Estudando a utilização do teste de etanol durante o armazenamento de sementes de repolho e avaliando a qualidade de sementes de azévem, Kodde e Groot (2015) e Cavalcante et al. (2017) respectivamente, observaram que o teste de etanol apresentava elevada sensibilidade para avaliar a deterioração das sementes, neste contexto demonstrando a eficiência do teste em diferentes espécies.

O teste de quantificação de etanol deve estar relacionado a dois fatores: integridade da membrana e atividade respiratória. Ambos os eventos estão intimamente relacionados a deterioração de sementes. Neste sentido Delouche e Baskin (1973) propuseram uma série de eventos durante a deterioração da semente, que leva à perda de vigor e germinação. 0 primeiro evento deste modelo é a deterioração da membrana. Em contraponto, a primeira atividade metabólica das sementes, concomitante à reidratação, é a respiração (BEWLEY; BLACK, 1994). Para a determinação do vigor, uma das alternativas é submeter as sementes à avaliação da atividade respiratória, a redução na germinação e no vigor se correlaciona positivamente com o aumento da atividade respiratória, pois tecidos mais degradados apresentam maior atividade respiratória do que tecidos íntegros (DODE et al., 2013).

Observando as condições do presente teste, no qual a semente encontra-se totalmente submersa em água destilada, ou seja, em ausência de $\mathrm{O}_{2}$, a produção de etanol oriunda da respiração anaeróbica ou via fermentativa, está relacionada à atividade respiratória. Como a produção de etanol provavelmente é relacionada com a perda da integridade da membrana mitocondrial, um ensaio baseada na 
quantificação de etanol tem um bom potencial como teste para avaliar o nível de vigor das sementes, além de ser um teste simples e rápido (BUCKLEY et al., 2006, BUCKLEY; HUANG, 2011).

Assim baseado nos resultados obtidos no presente trabalho, onde as sementes com maior nível de vigor (Tabela 1), apresentaram correlação negativa com a produção de etanol (Tabela 2), indica que sementes com menor nível de vigor, ou seja, com maior nível de deterioração das membranas e maior atividade respiratória tendem a apresentam maior liberação de etanol. Por outro lado, sementes com menor nível de deterioração, ou seja, com maior vigor tendem a apresentar menor atividade respiratória, ocasionando em condições de anaerobiose menor produção de etanol. Em estudos avaliando a produção de etanol em sementes de repolho, Rutzke et al. (2008) mostraram que os principais fatores que interferem na produção de etanol são a concentração de oxigênio e a integridade da semente.

Baseado nos resultados e discussão anterior, a quantificação da produção de etanol constitui-se em uma importante ferramenta na avaliação da qualidade fisiológica de lotes de sementes. $O$ teste do etanol demonstra elevada sensibilidade para avaliação do potencial fisiológico de sementes.

CONCLUSÃO

O teste de quantificação de etanol é eficiente na avaliação de vigor em sementes de quinoa.

A massa de $1 \mathrm{~g}$ de sementes é a mais indicada para condução do teste.

\section{REFERÊNCIAS}

BAALBAKI, R. BAALBAKI, R.; ELIAS, S.; MARCOSFILHO, J.; MCDONALD, M.B. Seed vigor testing handbook. Ithaca: Association of Official Seed Analysts, 2009.

BORGES, C.T.; SOARES, V. N.; MENEGHELLO, G. E.; COSTA, C. J.; GADOTTI, G. I.; VILLELA, F. A.; CASTELLANOS, C. I. S. Estabelecimento de metodologia para teste de germinação de Chenopodium Quinoa Willd. Informativo ABRATES, Curitiba, v. 23, n. 2, 2015.

BEWLEY, J.D.; BLACK, M. Seed physiology of development and germination. 2. ed. New York: Plenum, 1994.

BUCKLEY, W. T.; HUANG, J. An ethanol-based seed vigour assay for canola. Seed Science and
Technology, v.39, n.2, p.510-526, 2011. https://doi.org/10.15258/sst.2011.39.2.22

CAVALCANTE, J.; TERNUS, R. M.; ALMEIDA, T.L.; REOLON, F.; ARAUJO, A.S.; GADOTTI, G. I.; MORAES, D.M.M. Ethanol vigor test to assess physiological quality of annual ryegrass seeds. Espacios, v. 38, p. 10-18, 2017.

DELOUCHE, J.C.; BASKIN, C.C. Accelerated aging techniques for predicting the relative storability of seed lots. Seed Science and Technology, v.1, n.2, p.427-452, 1973.

DODE, J.S.; MENEGHELLO, G.E.; TIMM, F.C.; MORAES, D.M.; PESKE, S.T. Teste de respiração em sementes de soja para avaliação da qualidade fisiológica. Ciência Rural, v.43, n.2, p.193-198, 2013. DOI: http://dx.doi.org/10.1590/s0103$\underline{84782013000200001}$

KODDE, J.; BUCKLEY, W.T.; GROOT, C.C.; RETIERRE, M.; ZAMORA, A.M.V.; GROOT, S.P.C.. A fast ethanol assay to detect seed deterioration. Seed Science Research, v.22, n.1, p.55-62, 2012. https://doi.org/10.1017/S0960258511000274

KODDE, J; GROOTE, S.P.C. Ethanol degradation a simple and sensitive indicator for seed aging. Disponivel em: http://www.researchgate.net/publication/28003 9377 Ethanol degradation a simple and sensit ive indicator for seed aging. Acesso em: 19 de set. 2015.

MAGUIRE, J.D. Speed of germination aid in selection and evaluation for seedling emergence and vigor. Crop Science, v.2, n.2, p.176-77, 1962. https://doi.org/10.2135/cropsci1962.0011183X0 $\underline{00200020033 x}$

MARCOS FILHO, J. Testes de vigor: importância e utilização. In: KRZYZANOWSKI, F.C.; VIEIRA, R.D.; FRANÇA NETO, J.B. Vigor de sementes: conceitos e testes. Londrina: ABRATES, 1999. p.1.1-.121.

SÁ BRAZ, M.R.; ROSSETO, S.A.V. Correlação entre testes para avaliação da qualidade de sementes de girassol e emergência das plântulas em campo. Ciência Rural, v.39, n.7, p.2004-2009, $2009 . \quad$ https://doi.org/10.1590/S0103$\underline{84782009005000146}$ 
TAIZ, L.; ZEIGER, E. Fisiologia vegetal. Porto Alegre: Artmed, 2009.

RUTZKE, C.F.J; TAYLOR, A.G.; OBENDORF,R.L. Influence of aging, oxygen, and moisture on ethanol production from cabbage seeds. J. Amer. Soc. Hort. Sci., v.133, n.1, p.158-164. 2008.

TORRES, S. B.; DANTAS, A. H.; PEREIRA, M. F. S.; BENEDITO, C. P.; SILVA, F.H.A. Deterioração controlada em sementes de coentro. Revista Brasileira de Sementes, v. 34, n. 2, p. 319-326, $2012 . \quad$ https://doi.org/10.1590/S0101$\underline{31222012000200018}$

WENDT, L.; MALAVASI, M.M.; DRANSKI, J.A.L.; MALAVASI, U.C.; GOMES JUNIOR, F.C. Relação entre testes de vigor com a emergência a campo em sementes de soja. Agraria, v.12, n.2, p. 166171 , 2017.

https://doi.org/10.5039/agraria.v12i2a5435

WOODSTOCK,L.W; TAYLORSON, L.B. Ethanol and acetaldehyde in imbibing soybean seeds in relationto deterioration. Plant Physiol., v.67, p. 424-428, 1981.

https://doi.org/10.1104/pp.67.3.424

Recebido para publicação em 12/10/2017

Revisado em 28/06/2018

Aceito em 04/07/2018 